\title{
Conscious sedation technique for anxious children
}

\author{
An RCT pilot study to test the effects of intravenous midazolam as a conscious sedation technique for anxious \\ children requiring dental treatment - an alternative to general anaesthesia
}

P. A. Averley, I. Lane, J. Sykes, N. M. Girdler, N. Steen and S. Bond Br Dent J 2004; 197: 553-558

\section{Aim}

To add to the evidence base for acceptable and effective paediatric conscious sedation techniques in dental primary care.

\section{Objectives}

To compare three conscious sedation techniques for primary care as an alternative to dental general anaesthesia (DGA) in children. To assess the feasibility and practicality of running the trial in general dental practice. To form the basis for sample size calculations and assess scales of measurement.

\section{Design}

Single centre, randomised control trial (RCT).

\section{Setting}

Queensway Anxiety Management Clinic (QAMC). A primary carebased general and referral dental practice for the management of anxious patients.

\section{Subjects, materials and methods}

Sixty-five children too anxious for management with relative analgesia, requiring invasive dental procedure for which dental general anaesthesia (DGA) will be required if an alternative cannot be found.

\section{Interventions}

Group $1(n=20)$ - A combination of inhaled medical air and titrated intravenous midazolam. Group $2(n=22)$ - A combination of inhaled 40\% nitrous oxide in oxygen and titrated intravenous midazolam. Group $3(n=23)-$ A combination of an inhaled mixture of $0.3 \%$ sevoflurane and $40 \%$ nitrous oxide in oxygen with titrated intravenous midazolam.

\section{Main outcome measures}

Successful completion of the intended dental treatment with a child who is co-operative and responsive to verbal commands.

\section{Results}

Fifty per cent (ten children) successfully completed treatment in Group 1, 73\% (16 children) in Group 2 and 83\% (19 children) in Group 3. This difference was not significant at a $5 \%$ level $\left(\chi^{2}=5.53, \mathrm{df}=2, P=0.07\right)$ Of the total of 20 failures, eight children in Group 1 and one child in Group 2 were successfully treated with the addition of sevoflurane and nitrous oxide in oxygen. Only two children required referral to a hospital setting for DGA and the remaining nine children were managed with an alternative conscious sedation technique.

\section{Conclusion}

This pilot shows that intravenous midazolam especially in combination with the addition of inhaled nitrous oxide or sevoflurane and nitrous oxide were promising safe and effective techniques, sufficient to justify progression to a definitive RCT with appropriate methods.

\section{COMMENT}

The management of anxious paediatric dental patients is an area of great controversy. Many individuals have entrenched views, which are not necessarily supported by scientific evidence. The decline in the availability of general anaesthesia means that alternative management strategies must be found to allow these patients to be managed within the primary care environment.

The first line technique for the management of the anxious child is inhalation sedation with a titrated dose of nitrous oxide in oxygen. This technique, whilst highly successful, is not adequate for all children.

This paper aims to find a technique that will allow those children who have either not coped with inhalation sedation, or are felt to be unsuitable for inhalation sedation to have dental treatment successfully completed under conscious sedation.

The techniques described include the use of intravenous midazolam with or without the addition of inhalation sedation with nitrous oxide or nitrous oxide and sevoflurane. These techniques fulfil the General Dental Council Guidance. The GDC definition of sedation does not prescribe the number of agents used. The guidance does state that normally only a single agent will be required, and that use of intravenous sedation in children should be the exception rather than the rule. In this study the success of the combinations of drugs are higher than that of the single agent.

The conclusion of this pilot study is quite correct to suggest that a full randomised control trial has to be carried out (and the authors indicate that this is being completed).

This first stage in assessment of this strategy for managing anxious children is welcome as it points to the development of evidence-based practice in the field of conscious sedation in dentistry. The authors do, quite correctly, point out that conscious sedation techniques must only be used by those who are adequately trained and experienced in the particular technique (as required by all current guidance).

Although very encouraging, the evidence in this paper must not be used to devalue the role of the conventional technique of inhalation sedation for those patients for whom it is indicated and successful. There is no justification for the use of an 'advanced' technique when a basic one is appropriate. I would encourage readers to look out for the full study paper when it appears.

N. D. Robb, Senior Lecturer in Sedation in Relation to Dentistry, Glasgow Dental Hospital and School doi:10.1038/sj.bdj.4811806

\section{IN BRIEF}

- Intravenous sedation in combination with inhaled nitrous oxide or inhaled nitrous oxide and sevoflurane provides a safe alternative to general anaesthesia in anxious paediatric dental patients.

- With the appropriate staff, training and facilities, these conscious sedation techniques may be safely used in a 'non hospital setting' without the risk of 'deep sedation' or anaesthesia.

- It is essential that future research in the field of paediatric conscious sedation be carried out in the United Kingdom. 